Check for updates

Cite this: Mater. Adv., 2021, 2, 4859

Received 1st April 2021, Accepted 4th June 2021

DOI: $10.1039 / \mathrm{d} 1 \mathrm{ma} 00296 \mathrm{a}$

rsc.li/materials-advances

\section{Asymmetric $D-A-D^{\prime}$ Scaffold inducing distinct mechanochromic luminescence $\dagger$}

\author{
Huifang Yang, ${ }^{a}$ Zhiyuan Fu, ${ }^{\mathrm{b}}$ Sai Wang, ${ }^{\mathrm{a}}$ Haichao Liu, ${ }^{\mathrm{c}}$ Jianwei Zhou, ${ }^{a}$ \\ Kai Wang, (D) ${ }^{\mathrm{b}}$ Xiugang Wu, ${ }^{* a}$ Bing Yang, (D) ${ }^{\mathrm{c}}$ Bo Zou (D) ${ }^{\mathrm{b}}$ and Weiguo Zhu (D) *a
}

\begin{abstract}
Mechanochromic luminescence $(\mathrm{MCL})$ materials have a high requirement in high-tech applications such as pressure sensors and data storage. Nevertheless, the diversity of these emitters is rather restricted owing to the lack of clear molecular structure design guidelines, and it is imperative to develop creative multi-stimulus responsive compounds with polychromatic emission under different types of mechanical forces. In order to overcome difficulties of a single molecule exhibiting obvious MCL responses towards mechanical forces, in this study, a powerful approach of distinct multi-stimulus responsive $\mathrm{MCL}$ materials is to integrate the distinguished ability of donor units $\left(D^{\prime}\right)$, i.e. stronger phenothiazine (PTZ) and weaker bis(4-(tert-butyl)phenyl)amine (tDPA) compared with $9 \mathrm{H}$-carbazole, directly into the same donor-acceptor (D-A) scaffold of 2-(4-(carbazol-9-yl)phenyl)-isoindoline-1,3-dione (CzPL) and hence obtaining CzPL-PTZ and CzPL-tDPA. Distinct MCL were primarily found for the parent CzPL-PTZ and CzPL-tDPA crystals under tensile force, and anisotropic and isotropic pressures. Interestingly, both crystals show an abnormal blue-shifted emission under crushing force. When under anisotropic pressure, the CzPL-PTZ ground sample further displays blue-shifted emission, but the CzPL-tDPA ground sample displays red-shifted emission. In contrast, both parent crystals show a similarly redshifted and weakened emission under increasing isotropic pressure. Furthermore, these emissions exhibit a linear relationship between wavelength and pressure. More importantly, crystal CzPL-PTZ can completely return to the initial state once decompressed due to the reversible 3D short-range intermolecular interaction network; in contrast, the crystal CzPL-tDPA cannot fully recover to its original state after removing pressure due to the lattice vibration under a high pressure. The interesting and distinct MCL behaviors of CzPL-PTZ and CzPL-tDPA indicate that the D-A-D' molecular design should provide pragmatic insights towards MCL materials.
\end{abstract}

\section{Introduction}

Mechanochromic luminescence (MCL) materials with polychromatic switches can exhibit distinctly variational emission when subjected to external mechanical stimuli (e.g., smashing, grinding, stretching, or hydrostatic pressure) ${ }^{1-6}$ Such materials have been widely studied due to their potential applications in

\footnotetext{
${ }^{a}$ School of Materials Science and Engineering, Jiangsu Collaboration Innovation Center of Photovoltaic Science and Engineering, Jiangsu Engineering Laboratory of Light-Electricity-Heat Energy-Converting Materials and Applications, National Experimental Demonstration Center for Materials Science and Engineering, Changzhou University, Changzhou 213164, P. R. China. E-mail: zhuwg18@126.com

${ }^{b}$ State Key Laboratory of Superhard Materials, College of Physics, Jilin University, Changchun 130012, P. R. China

${ }^{c}$ State Key Laboratory of Supramolecular Structure and Materials, College of Chemistry, Jilin University, Changchun 130012, P. R. China

$\dagger$ Electronic supplementary information (ESI) available. CCDC 2071819 and 2071820. For ESI and crystallographic data in CIF or other electronic format see DOI: $10.1039 / \mathrm{d} 1 \mathrm{ma} 00296 \mathrm{a}$
}

optical data storage, pressure sensors, anti-counterfeiting inks, and optoelectronic devices. ${ }^{7-24}$ In general, MCL can be realized by chemical structure change (e.g., chemical bond destruction and recombination $)^{25,26}$ or physical structure change (e.g., phase transition ${ }^{27-29}$ and conformational transition ${ }^{30-32}$ ). The emission properties of MCL materials largely depend on molecular stacking modes, which are closely related to the molecular conformation and noncovalent interactions. ${ }^{33-35}$ External forces can change the molecular stacking mode, subsequently managing the emission colors and intensities. ${ }^{10-17,36,37}$

Most MCL materials show reversible conversion luminescence under only one mechanical force. ${ }^{38-42}$ However, materials with distinct MCL responses towards different mechanical stimuli are very rare. For instance, Wang certificated that boron-diketonate crystals exhibited unique emissions under different mechanical stimuli, with a blue-shift after crushing and a red-shift under grinding and compressing. ${ }^{43}$ Yamaguchi reported that a tetrathiazolylthiophene fluorophore displayed distinctly emissive responses to mechanical grinding and hydrostatic pressures. ${ }^{44}$ 
Liu found that a donor-acceptor charge-transfer cocrystal CT-R also exhibited differently emissive responses to anisotropic grinding and isotropic compressing. ${ }^{23}$ From the viewpoint of applications, materials with different MCL responses seem to be more attractive. Nevertheless, the diversity of these emitters was still rather restricted owing to the lack of clear molecular structure design guidelines, and it is imperative to develop creative multistimulus responsive MCL materials with polychromatic emission under different types of mechanical forces.

Herein, two novel molecules, namely 2-(4-(9H-carbazole-9-yl)phenyl)-5-(10H-phenothiazine-10-yl)isoindoline-1,3-dione (CzPL-PTZ) and 2-(4-(9H-carbazole-9-yl) phenyl)-5-(bis(4-(tert-butyl)phenyl)amino)isoindoline-1,3-dione (CzPL-tDPA), were designed and synthesized by integrating distinguishing ability of electrondonating units $\left(\mathrm{D}^{\prime}\right)$ into the same donor-acceptor $(\mathrm{D}-\mathrm{A})$ scaffold of 2-(4-(carbazol-9-yl)phenyl)-isoindoline-1,3-dione (CzPL). In both molecules, phthalimide is chosen as an electron-withdrawing acceptor (A) owing to its excellent stability, mechanical toughness, and rigid structure. ${ }^{45} 9 \mathrm{H}$-carbazole $(\mathrm{Cz})$ is employed as an endcapping electron-donating group due to its planar $\pi$ conjugation and good charge transport ability. Pseudo-planar phenothiazine (PTZ) and propeller-like bis(4-(tert-butyl)phenyl)amine (tDPA) are used as the $\mathrm{D}^{\prime}$ units make the molecular packing can be adjusted, eventually resulting in exhibit distinct MCL of CzPL-PTZ and CzPL-tDPA. Overall, the sturdy intramolecular hydrogen bonds in phthalimide should rigidify the molecular conformation and suppress the nonradiative decay. ${ }^{46-48}$ Moreover, the abundant hydrogen bonds between the CzPL unit and distinguishing stereochemical structures are available for the resulting molecules to form different three-dimensional (3D) networks, and different multi-stimuli responsive MCL materials can be obtained by adjusting the electron donating ability of $\mathrm{D}^{\prime}$ units. Our study provides a new design strategy of asymmetric $\mathrm{D}-\mathrm{A}-\mathrm{D}^{\prime}$ to develop novel MCL materials.

\section{Results and discussion}

\subsection{Synthesis and characterization}

Fig. 1 shows the chemical structures of CzPL-PTZ and CzPL-tDPA. Their synthetic routes are shown in Scheme S1 ESI. $\dagger$ They were synthesized by the Buchwald-Hartwig C-N cross-coupling reaction between CzPL-Br and PTZ or tDPA in moderate yields. Subsequently, they were purified via vacuum sublimation. Their molecular structures were fully characterized by ${ }^{1} \mathrm{H},{ }^{13} \mathrm{C}$ NMR spectroscopy and TOF-MS (see ESI $\dagger$ ). Thermogravimetric analysis (TGA) measurements indicate that both CzPL-PTZ and CzPL-tDPA have good thermal stability with high decomposition temperatures

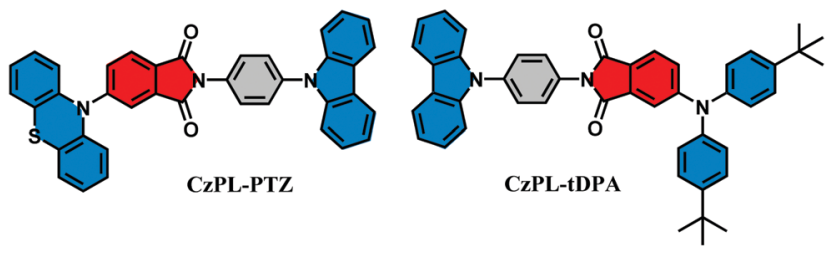

Fig. 1 Chemical structures of CzPL-PTZ and CzPL-tDPA.
( $T_{\mathrm{d}}$, corresponding to $5 \%$ weight loss) of $418{ }^{\circ} \mathrm{C}$ and $428{ }^{\circ} \mathrm{C}$, respectively (Fig. S1 in $\mathrm{ESI} \dagger$ ).

\subsection{Crystal structures}

Since molecular packing plays a key role in MCL, the crystal structures of CzPL-PTZ and CzPL-tDPA were deeply investigated, which might give important insights into the MCL mechanism. The yellow-green (533 nm) and blue-green (498 nm) photoluminescent (PL) peaks are observed for the CzPL-PTZ and CzPL-tDPA parent crystals made via vacuum sublimation (Fig. S2 in ESI $\dagger$ ), respectively. Both parent crystals possess quantum yield $\left(\Phi_{\mathrm{f}}\right)$ values of $39 \%$ and $66 \%$ correspond to fluorescence lifetimes $(\langle\tau\rangle)$ of 12.07 ns and 16.56 ns for CzPL-PTZ and CzPL-tDPA (Fig. S3 and Table S1 in ESI $\dagger$ ). Fig. 2 shows the crystal structures and intermolecular interactions of the CzPL-PTZ and CzPL-tDPA crystals measured by single crystal X-ray diffraction (XRD) experiments. Table S2 (ESI $\dagger$ ) summarizes the detailed data of these crystal structures. The results indicate that CzPL-PTZ adopts a quasi-axial conformation and a regular head-to-tail arrangement throughout some $\mathrm{C}-\mathrm{H} \cdots \pi, \mathrm{C}-\mathrm{H} \cdots \mathrm{N}, \mathrm{C}-\mathrm{H} \cdots \mathrm{O}, \mathrm{C}-\mathrm{H} \cdots \mathrm{S}$, and weak $\pi \cdots \pi$ interactions (Fig. 2a). In contrast, CzPL-tDPA features a head-to-tail arrangement but $Z$-shaped packing between adjacent molecules (Fig. 2b). These multiple intermolecular interactions can restrict the intramolecular motions and vibrations for both crystals, which is beneficial to the increase the $\Phi_{\mathrm{f}}$ values. Moreover, the twisty molecular configurations with large dihedral angles between phenylene and phthalimide/ carbazolyl (Fig. S4 in ESI $\dagger$ ) and the $\pi \cdots \pi$ distance over $3.518 \AA$ between anti-parallel molecules make both crystals present a weak $\pi \cdots \pi$ stacking interaction, in which the aggregation caused quenching (ACQ) can be efficiently suppressed. Their densities of $1.386 \mathrm{~g}$ $\mathrm{cm}^{-3}$ and $1.224 \mathrm{~g} \mathrm{~cm}^{-3}$ for CzPL-PTZ and CzPL-tDPA crystals indicate that the triclinic CzPL-PTZ crystal has much tighter stacking than the monoclinic CzPL-tDPA crystal. Interestingly, both crystals exhibit multiple intermolecular interactions observed from the $a, b, c$ axes, and form a huge 3D short-range intermolecular interaction network (Fig. S5 in ESI $\dagger$ ). Such short-range 3D networks with weak intermolecular interactions should be easy to be deformed and get distinct emissive responses under different mechanical forces (e.g., crushing, grinding, or hydrostatic pressure).

\subsection{Photophysical properties}

The UV-Visible and PL spectra of CzPL-PTZ and CzPL-tDPA were obtained in their solutions with different solvents and neat films. Two typical absorption bands were observed in Fig. S6 (ESI $\dagger$ ), which contain a high-lying band of absorption in the range of $300-350 \mathrm{~nm}$ and a low-lying band of absorption in the range of 350-450 $\mathrm{nm}$. The low-lying bands of absorption are ascribed to the intramolecular charge transfer (ICT) absorption. ${ }^{49-51}$ Fig. S6 (ESI $\dagger$ ) also shows the PL spectra of CzPL-PTZ and CzPL-tDPA in different solvents $\left(1.0 \times 10^{-5} \mathrm{M}\right)$. Dual emissions were observed for CzPL-PTZ in toluene (Tol), chloroform (CF), and tetrahydrofuran (THF), which were assigned to the dual conformers of PTZ (Fig. S6a in ESI $\dagger$ ). ${ }^{52}$ The excitation spectra of CzPL-PTZ at $480 \mathrm{~nm}$ and $560 \mathrm{~nm}$ in the CF solvent $\left(1.0 \times 10^{-5} \mathrm{M}\right)$ with different spectra indicate that the two emission bands at 

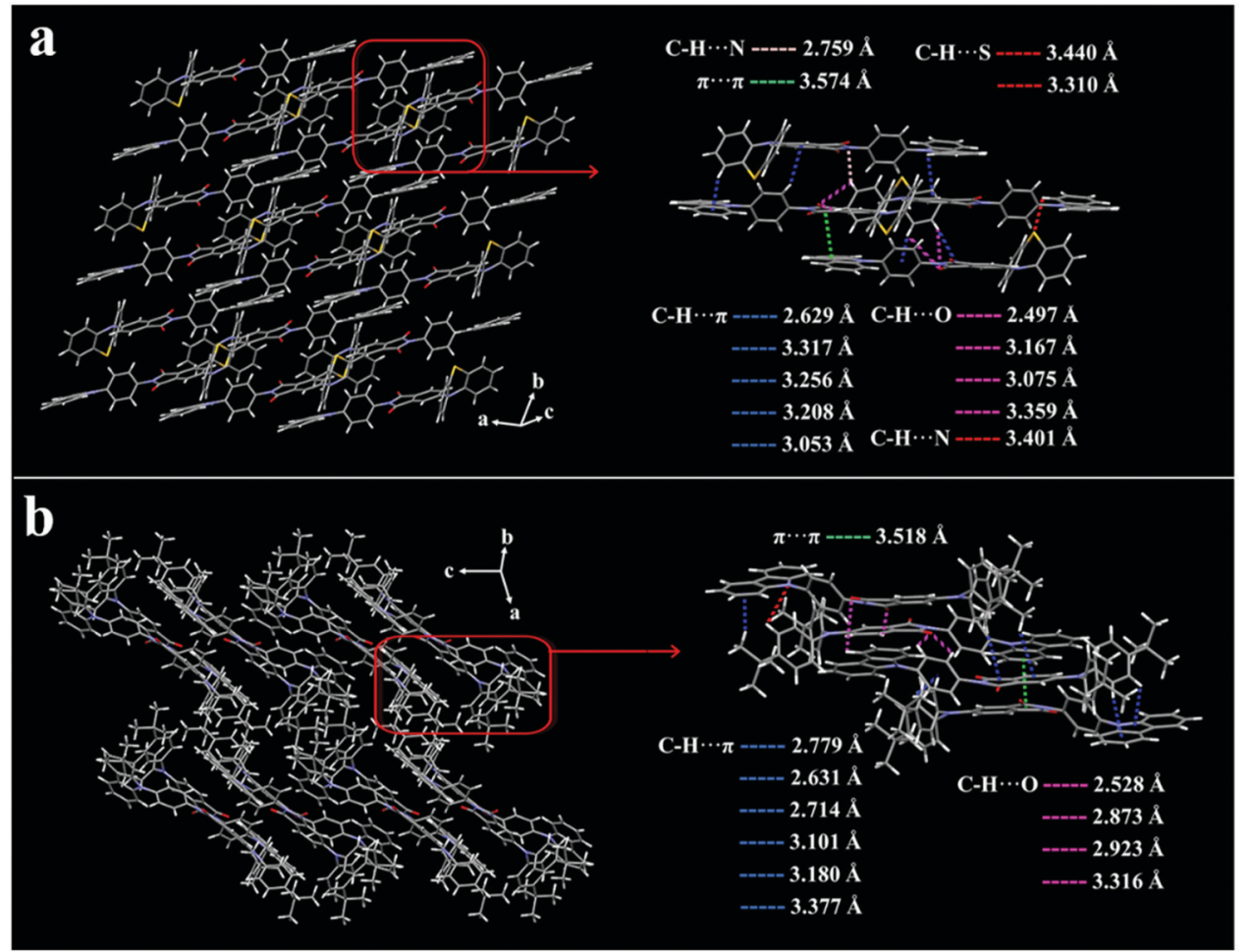

Fig. 2 Crystal structures and intermolecular interactions of the (a) CzPL-PTZ and (b) CzPL-tDPA crystals.

$480 \mathrm{~nm}$ and $560 \mathrm{~nm}$ come from different excited states, i.e. locally excited (LE) and CT states (Fig. S7 in ESI $\dagger$ ). ${ }^{52-58}$ In contrast, there is only single emission peak for CzPL-tDPA in different solutions (Fig. S6b in ESI $\dagger$ ). Both molecules show obvious solvent dependence in different polar solvents owing to their ICT effects. In addition, different PL spectra are observed for both molecules under two solid states. Due to the special quasi-axial conformation of the PTZ unit (bending to one side, small steric hindrance), the linear CzPL-PTZ molecules are easy to pile up in the long-range order and exhibit relatively strong intermolecular interactions. In contrast, CzPL-tDPA can easily form a large twisty type between tDPA and CzPL, which results in a relatively weak intermolecular interaction owing to the large steric hindrance of tert-butyl. Therefore, CzPL-PTZ displayed more red-shifted luminescence than CzPL-tDPA in crystal and film states (Fig. S2 and S8 in ESI $\dagger$ ). ${ }^{56}$

\subsection{Theoretical calculations}

Density functional theory (DFT) discloses that the asymmetric D-A-D' strategy has an impact on the lowest-lying electronic transition. The electronic structures of CzPL-PTZ and CzPL-tDPA are optimized at the ground state $\left(\mathrm{S}_{0}\right)$ in the gas state using Gaussian 09W program at the B3LYP/6-31G (d)/LanL2DZ level. ${ }^{59,60}$ As shown in Fig. S9 (ESI $\dagger$ ), the lowest unoccupied molecular orbitals (LUMOs) of CzPL-PTZ and CzPL-tDPA are mainly located on electron deficient phthalimide moieties, and the highest occupied molecular orbital (HOMO) of CzPL-PTZ is dominantly distributed over the electron donor phenothiazine moieties. Since carbazole has stronger electron donating ability than bis (4-(tert-butyl) phenyl) amine, the HOMO of CzPL-tDPA is mainly distributed in the carbazole unit. ${ }^{61}$ Clearly, electron-donating $\mathrm{D}^{\prime}$ units with distinguishing ability led to distinction in the molecular level and hence influence the MCL properties in the aggregation state. In addition, the calculated HOMO and LUMO energy levels are $-5.28 /-2.63 \mathrm{eV}$ and $-5.18 /-2.08 \mathrm{eV}$ for CzPL-PTZ and CzPL-tDPA, respectively. The more effective spatial separation of HOMO and LUMO in the CzPLPTZ compound compared with CzPL-tDPA should demonstrate stronger ICT nature. ${ }^{62}$

\subsection{Electrochemical characterization}

Electrochemical properties of CzPL-PTZ and CzPL-tDPA were investigated by cyclic voltammetry (CV) adopting ferrocene as a reference standard in anhydrous $\mathrm{CH}_{3} \mathrm{CN}$ solutions. The recorded CV curves are shown in Fig. S10 (ESI $\dagger$ ) and their electrochemical data are listed in Table S4 (see ESI $\dagger$ ); CzPL-PTZ and CzPL-tDPA present reversible oxidation process in CV curves. According to the onset voltages of the oxidation curves, their HOMO levels are determined to be $-\mathbf{5 . 1 6}$ and $-5.41 \mathrm{eV}$ for CzPL-PTZ and CzPLtDPA, respectively. The lowest unoccupied molecular orbital (LUMO) energy level of CzPL-PTZ and CzPL-tDPA can be calculated using $E_{\text {HOMO }}$ and optical band gap $\left(E_{\mathrm{g}}\right)$, which are -2.04 and $-2.48 \mathrm{eV}$, respectively. ${ }^{63,64}$ In addition, the calculated HOMO and LUMO energy levels for CzPL-PTZ and CzPL-tDPA are $-5.28 /-2.63 \mathrm{eV}$ and $-5.18 /-1.18 \mathrm{eV}$, respectively, which are reasonable deviations from the experimental data.

\subsection{Mechanochromic luminescence properties}

The MCL properties of the CzPL-PTZ and CzPL-tDPA crystals were studied under different forces, such as tensile force, 
anisotropic, and isotropic pressures. Fig. 3a and b show the PL spectra of CzPL-PTZ and CzPL-tDPA in different solid states, respectively. Here, the crushed and ground samples were obtained under crushing using a stainless-steel spoon and ground for $30 \mathrm{~min}$ in a mortar. In addition, the PL spectra of CzPL-PTZ and CzPL-tDPA ground samples were recorded at room temperature after different retention time periods. It can be seen from the Fig. S12 (ESI $\dagger$ ) that there is almost no change in their PL spectra, which indicates that the two ground samples are very stable at room temperature. The fumed samples were obtained under fuming dichloromethane (DCM) vapor for $10 \mathrm{~min}$. It was found that both crushed crystals exhibited scarce blue-shifted emissions in comparison with their corresponding parent crystals. It implies that a proper splitting of the parent crystals can greatly disturb the ordered molecular arrangement on the surface, thus resulting in a depressed conjugated system and blue-shifted emission. ${ }^{42}$ More surprisingly, the $\Phi_{\mathrm{f}}$ values of the crushed crystals of CzPL-PTZ (48\%) and CzPL-tDPA (91\%) greatly improved in comparison with those of their corresponding parent crystals of CzPL-PTZ (39\%) and CzPL-tDPA $(66 \%)$ (Table S6 in ESI $\dagger){ }^{65}$

When the crushed crystals are further ground by a mortar, the resulting ground sample of CzPL-tDPA shows bathochromic-shift emission like most of the reported MCL materials. ${ }^{13,14,27-31}$ However, the ground sample of CzPL-PTZ shows a rare hypochromicshift emission. ${ }^{21,23,24,66}$ A broad emission band peak at $501 \mathrm{~nm}$ is observed in the CzPL-PTZ ground sample, which is blue-shifted by $18 \mathrm{~nm}$ in comparison with that of the CzPL-PTZ crushed crystal $(519 \mathrm{~nm})$. Under further fuming the ground samples with the polar solvent vapor of DCM for $10 \mathrm{~min}$, the resulting CzPL-PTZ fumed sample exhibited a red-shifted emission peaked at $520 \mathrm{~nm}$ (Fig. 3a). In contrast, the CzPL-tDPA ground sample presents a significantly red-shifted emission peak at $547 \mathrm{~nm}$ in comparison with that of the CzPL-tDPA crushed crystal (491 nm). The emission color was partially recovered after fumigation with DCM (Fig. 3b). Therefore, the alternative appendage of another electron-donating unit of PTZ and tDPA in the D-A unit of CzPL can make their crystals exhibit distinct MCL response in the above four states. Furthermore, the film emission of the two compounds was compared with that of crystals and ground samples (Fig. S13, ESI $\dagger$ ). The results show that the crystal and ground sample emission of the two compounds blue-shifted compared with that of the films. The conformation planarization and the closer molecular packing of CzPL-PTZ and CzPL-tDPA molecules in the films should be the origins for their red-shifted emission.

The powder X-ray diffraction (PXRD) diagrams of CzPL-PTZ and CzPL-tDPA in different states indicate that these various MCL responses are related to the change in the molecular packing structure, which are recorded in Fig.s S14a and b (ESI $\dagger$ ), respectively. The results show that CzPL-PTZ and CzPL-tDPA form different molecular stacking architectures over the crushing-grindingfuming treatment. These observations indicate that the strong and few diffraction peaks of CzPL-PTZ and CzPL-tDPA in the parent crystal state indicate that their crystals are polycrystalline. After crushing, the stacked polycrystals are split, the crystal size becomes smaller and the diffraction peaks increase. With further grinding, the PXRD pattern of the ground sample of CzPL-PTZ and CzPL-tDPA are composed of several blunt "peaks", indicating that they are mainly amorphous in the ground state or their crystallites are extremely small in size.
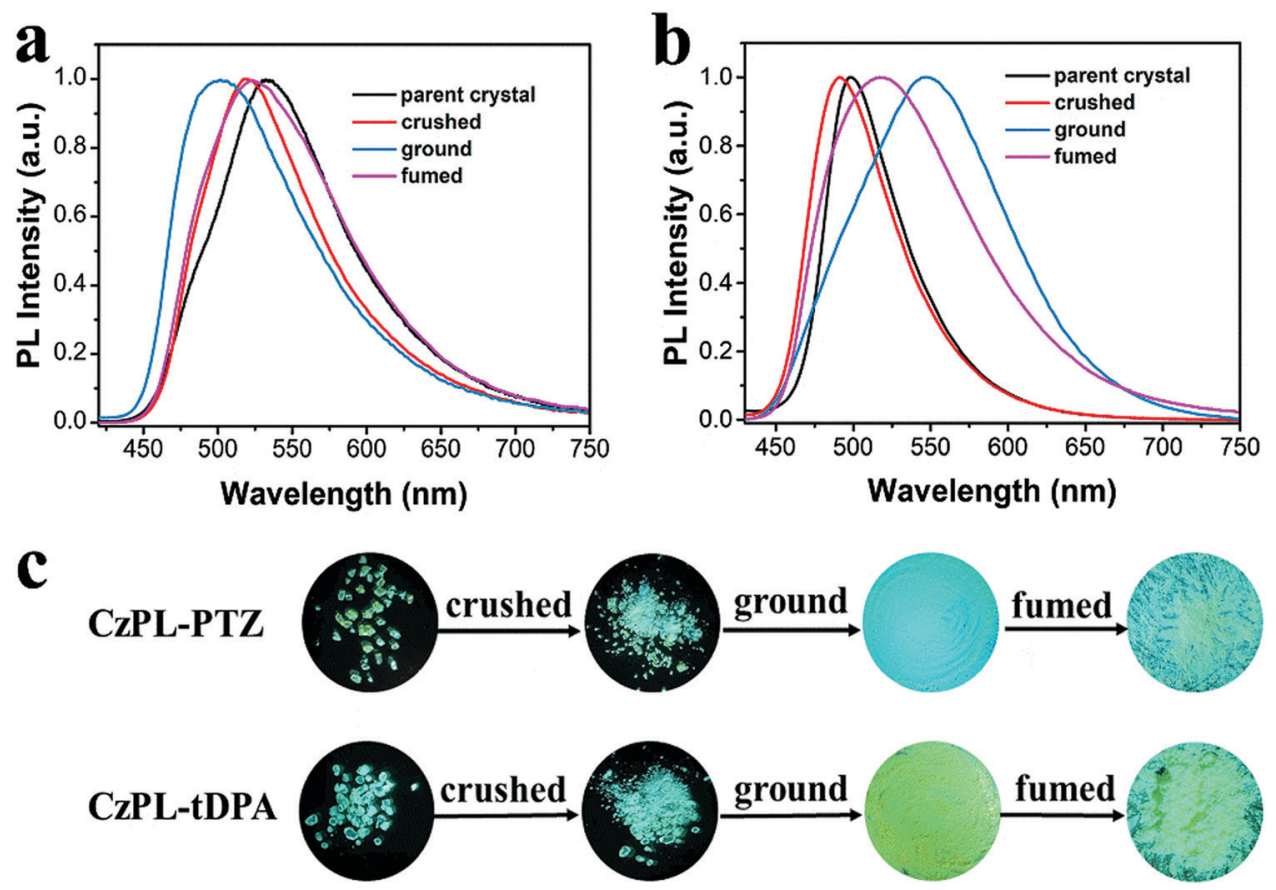

Fig. 3 PL spectra of (a) CzPL-PTZ and (b) CzPL-tDPA in their parent crystals, crushed crystals, ground samples, and fumed samples; (c) the photographs of CzPL-PTZ and CzPL-tDPA during the crushing-grinding-fuming processes under UV light irradiation at $365 \mathrm{~nm}$. 
Similarly, the ground samples of CzPL-PTZ and CzPL-tDPA after fumigation in dichloromethane (DCM) vapor for $10 \mathrm{~min}$ exhibit almost same PXRD patterns as those of single crystal simulation, indicating that the molecular arrangement in fumigated samples return to the crystalline state. There is no doubt that the molecular arrangement of CzPL-PTZ and CzPL-tDPA changed during mechanical grinding. For the crystal CzPL-PTZ, grinding may cause the aggregated molecules to slip, disrupt the 3D short range intermolecular interaction network to ongoing single molecules, which will lead to the blue-shift emission. ${ }^{44}$ The red-shift luminescence of CzPL-tDPA may be due to the different molecular arrangements such as $\pi$-stacked dimers have been formed after grinding. ${ }^{13,14,43}$

As mentioned above, the anisotropic pressure causes the CzPL-PTZ and CzPL-tDPA crystals to exhibit distinct MCL. To reveal the effects of isotropic pressure on emission, the PL spectra of both parent crystals under different hydrostatic pressures were further studied. As shown in Fig. 4, the CzPL-PTZ crystal exhibits a gradually red-shifted and weakened emission from 480 to $600 \mathrm{~nm}$, with the hydrostatic pressure increasing from ambient pressure to 10.31 GPa. Furthermore, its absorption is also observed to maintain a red-shifted trend (Fig. S15 in ESI $\dagger$ ). In contrast, when the hydrostatic pressure is returned to ambient pressure, its PL spectrum turns back to the original PL profile (Fig. 4b). Identically, CzPL-tDPA shows similarly red-shifted emission and absorption with the increase in the hydrostatic pressure (Fig. 5 and Fig. S16 in ESI $\dagger$ ). However, the PL spectrum of CzPL-tDPA just partly returns to the initial PL profile after the hydrostatic pressure is released to ambient pressure. More interestingly, whether it pressuring or decompressing the crystal, the emissive wavelength and energy have a direct good linear relationship to the hydrostatic pressure for both crystals (Fig. S17 and S18 in ESI $\dagger$ ). This emissive uniqueness endows this type of MCL materials with potential application in pressure sensors. ${ }^{5}$ Better emissive restorability of both crystals indicates that the molecular packing mode should not change substantially during a high-pressure process. Considering this point, the intense red-shift and weakened PL of both crystals may be attributed to the pressure-induced conformational planarization with increasing pressure, which leads to severe $\pi-\pi$ intermolecular interactions. ${ }^{67,68}$

According to the crystal structure analysis, there are weak $\pi-\pi$ interactions in both crystals. The $\pi-\pi$ distances of CzPL-PTZ and CzPL-tDPA are 3.574 $\AA$ and $3.518 \AA$, respectively, which are beyond the range of van der Waals interaction (Fig. 2b-d). The molecular distance between adjacent molecules should be shortened and $\pi-\pi$ interaction should be enhanced with increasing pressure.
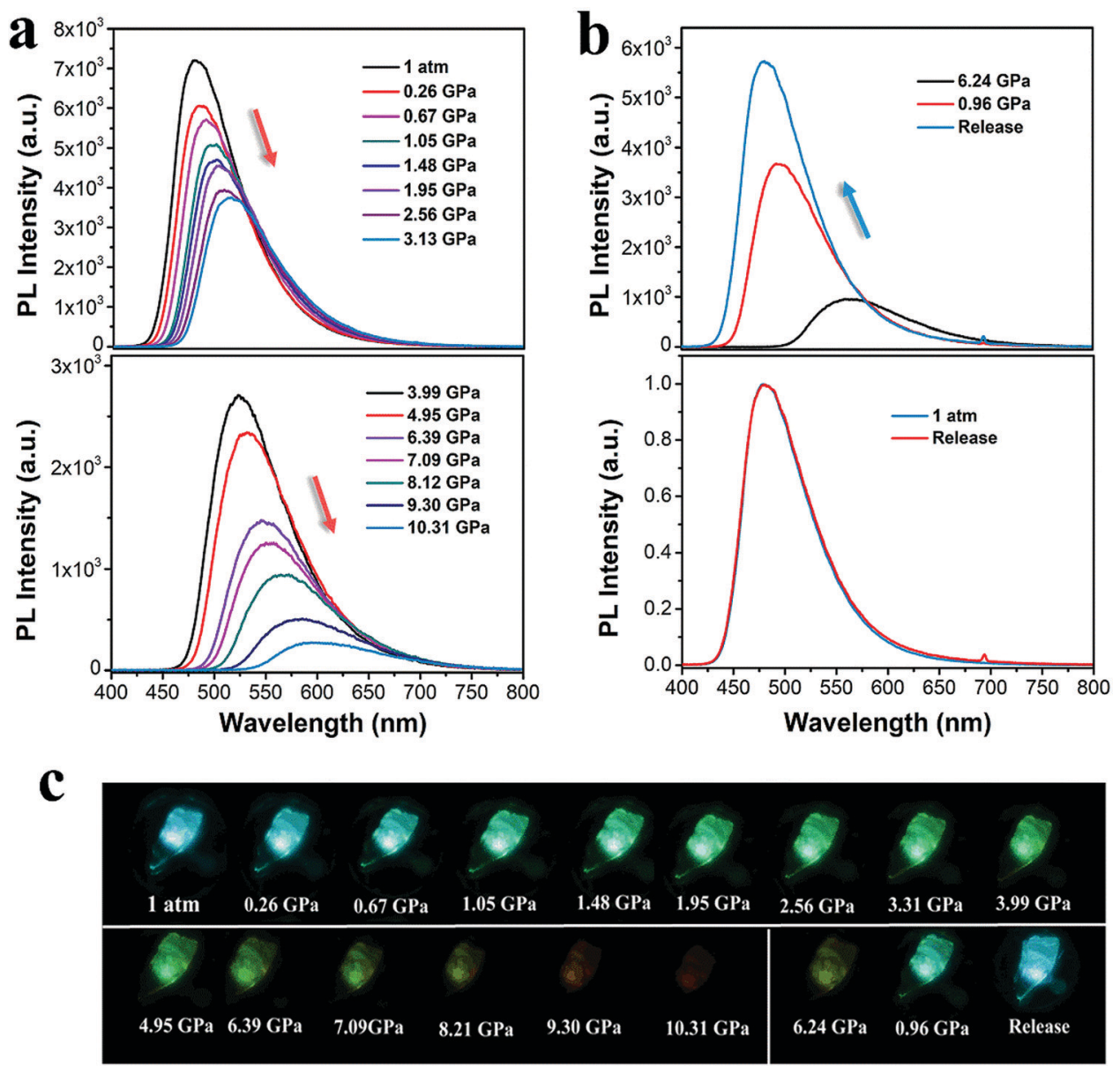

Fig. 4 (a) PL spectra of crystal CzPL-PTZ under the pressures from 1 atm to $10.31 \mathrm{GPa}$, (b) PL spectra of crystal CzPL-PTZ during the decompression processes, (c) the fluorescence images of the CzPL-PTZ crystal under photo excitation at $350 \mathrm{~nm}$ and different pressures. 

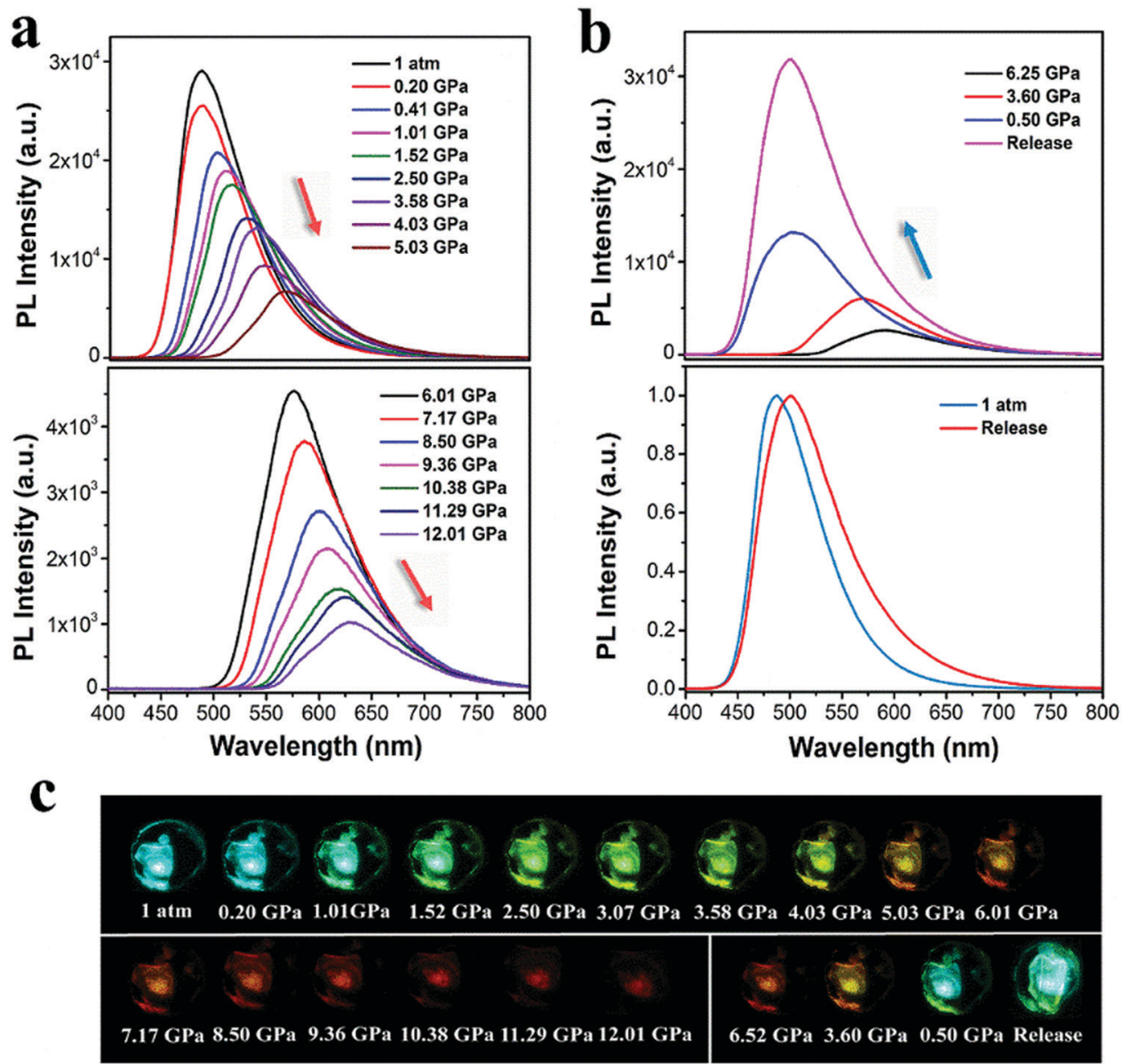

Fig. 5 (a) PL spectra of crystal CzPL-tDPA under the pressures from 1 atm to $12.01 \mathrm{GPa}$, (b) PL spectra of crystal CzPL-tDPA during the decompression processes, (c) the fluorescence images of the crystal CzPL-tDPA under photo excitation at $350 \mathrm{~nm}$ and different pressures.

As a result, red-shifted and weakened emissions are observed. The squashed CzPL-PTZ crystal can rebound to its original shape after decompressing. That is, its 3D intermolecular short-range interaction network undergoes a simple harmonic vibration within a certain pressure range during the compression-decompression process. Alternatively, the deformation of a 3D intermolecular short-range interaction network is reversible in this condition. Regarding the behavior of the CzPL-tDPA crystal under decompression, it might have to overcome some potential barrier to recover the $3 \mathrm{D}$ intermolecular short-range interaction network. Such an incomplete recovery is partially caused by the lattice vibration under high pressure. ${ }^{69}$

\section{Conclusion}

In summary, we have demonstrated a simple and effective molecular design strategy by integrating the distinguishing ability of electron-donating units $\left(\mathrm{D}^{\prime}\right)$ on the CzPL scaffold to obtain two novel MCL materials of CzPL-PTZ and CzPL-tDPA. The $\mathrm{D}^{\prime}$ units of pseudo-planar PTZ and propeller-like tDPA play an important role in the MCL properties of CzPL-PTZ and CzPL-tDPA parent crystals. As a result, both crushed crystals show an abnormal blue-shifted emission under tensile force in comparison with corresponding parent crystals; CzPL-PTZ crystals exhibit hypochromatic-shift, while CzPL-tDPA exhibit bathochromic shift when under anisotropic pressure. In contrast, both crystals show a red-shifted emission under increasing isotropic pressures. Significantly distinct MCL for both parent crystals under tensile force, anisotropic and isotropic pressures certify the effectiveness of the strategy D-A-D' scaffold. Interestingly, crystal CzPL-PTZ can completely return to the initial state after decompression, while crystal CzPL-tDPA cannot fully recover to its original state after removing pressure. More importantly, there is a linear relationship between emissive wavelength and pressure. The distinct and interesting MCL phenomena may have a potential application in pressure sensors and data storage.

\section{Conflicts of interest}

There are no conflicts to declare.

\section{Acknowledgements}

This work was supported by the National Natural Science Foundation of China (52073035, U1663229, and 21725304), the Top-notch Academic Programs Project (TAPP) for Polymeric 
Materials Science and Technology and the Priority Academic Program Development (PAPD) of Jiangsu Higher Education Institutions, the Jiangsu Provincial Talents Project of High-Level Innovation and Entrepreneurship, the Research Innovation Program for Postgraduate of Jiangsu Province (SJCX20_0973).

\section{References}

1 T. Seki, Y. Takamatsu and H. Ito, J. Am. Chem. Soc., 2016, 138, 6252-6260.

2 Y. J. Dong, B. Xu, J. B. Zhang, X. Tan, L. J. Wang, J. L. Chen, H. G. Lv, S. P. Wen, B. Li, L. Ye, B. Zou and W. J. Tian, Angew. Chem., Int. Ed., 2012, 51, 10782-10785.

3 L. Y. Bai, P. Bose, Q. Gao, Y. X. Li, R. Ganguly and Y. L. Zhao, J. Am. Chem. Soc., 2017, 139, 436-441.

4 B. J. Xu, Y. X. Mu, Z. Mao, Z. L. Xie, H. Z. Wu, Y. Zhang, C. J. Jin, Z. G. Chi, S. W. Liu, J. R. Xu, Y.-C. Wu, P.-Y. Lu, A. Lien and M. R. Bryce, Chem. Sci., 2016, 7, 2201-2206.

5 T. Ono, Y. Tsukiyama, A. Taema, H. Sato, H. Kiyooka, Y. Yamaguchi, A. Nagahashi, M. Nishiyama, Y. Akahama, Y. Ozawa, M. Abe and Y. Hisaeda, ChemPhotoChem, 2018, 2, 416-420.

6 Y. Sagara, T. Mutai, I. Yoshikawa and K. Araki, J. Am. Chem. Soc., 2007, 129, 1520-1521.

7 Z. G. Chi, X. Q. Zhang, B. J. Xu, X. Zhou, C. P. Ma, Y. Zhang, S. W. Liu and J. R. Xu, Chem. Soc. Rev., 2012, 41, 3878-3896.

8 P. Theato, B. S. Sumerlin, R. K. O'Reilly and T. H. Epps III, Stimuli responsive materials, Chem. Soc. Rev., 2013, 42, 7055-7056.

9 Y. Sagara, S. Yaman, M. Mitani, C. Weder and T. Kato, Adv. Mater., 2016, 28, 1073-1095.

10 C. Wang and Z. Li, Mater. Chem. Front., 2017, 1, 2174-2194.

11 S.-J. Yoon, J. W. Chung, J. Gierschner, K. S. Kim, M.-G. Choi, D. Kim and S.-Y. Park, J. Am. Chem. Soc., 2010, 132, 13675-13683.

12 Y.-B. Gong, P. Zhang, Y.-R. Gu, J.-Q. Wang, M.-M. Han, C. Chen, X.-J. Zhan, Z.-L. Xie, B. Zou, Q. Peng, Z.-G. Chi and Z. Li, Adv. Opt. Mater., 2018, 6, 1800198.

13 T. Ito, T. Saito, N. Oshima, N. Kitamura, S. Ishizaka, Y. Hinatsu, M. Wakeshima, H. Yukio, M. Kato, K. Tsuge and M. Sawamura, J. Am. Chem. Soc., 2008, 130, 10044-10045.

14 Q. K. Qi, J. Y. Qian, X. Tan, J. B. Zhang, L. J. Wang, B. Xu, B. Zou and W. J. Tian, Adv. Funct. Mater., 2015, 25, 4005-4010.

15 Y. Sagara and T. Kato, Angew. Chem., Int. Ed., 2008, 120, 5253-5256.

16 Y. Sagara, S. Yamane, T. Mutai, K. Araki and T. Kato, Adv. Funct. Mater., 2009, 19, 1869-1875.

17 Y. Sagara and T. Kato, Angew. Chem., Int. Ed., 2011, 50, 9128-9132.

18 F. Ciardelli, G. Ruggeri and A. Pucci, Chem. Soc. Rev., 2013, 42, 857-870.

19 K. M. Wiggins, J. N. Brantley and C. W. Bielawski, Chem. Soc. Rev., 2013, 42, 7130-7147.

20 A. Lavrenova, D.-W. Balkenende, Y. Sagara, S. Schrettl, Y. C. Simon and C. Weder, J. Am. Chem. Soc., 2017, 139, 4302-4305.

21 D. P. Yan, H. J. Yang, Q. Y. Meng, H. Y. Lin and M. Wei, Adv. Funct. Mater., 2014, 24, 587-594.
22 Y. Matsunaga and J.-S. Yang, Angew. Chem., Int. Ed., 2015, 54, 7985-7989.

23 Y. J. Liu, Q. X. Zeng, B. Zou, Y. Liu, B. Xu and W. J. Tian, Angew. Chem., Int. Ed., 2018, 57, 15670-15674.

24 H. C. Liu, Y. R. Gu, Y. X. Dai, K. Wang, S. T. Zhang, G. Chen, B. Zou and B. Yang, J. Am. Chem. Soc., 2020, 142, 1153-1158.

25 J. W. Chung, Y. You, H. S. Huh, B. K. An, S.-J. Yoon, S. Kim, H. Kim, S. W. Lee and S. Y. Park, J. Am. Chem. Soc., 2009, 131, 8163-8172.

26 Y. Wang, X. Tan, Y. M. Zhang, S. Zhu, I. Zhang, B. Yu, K. Wang, B. Yang, M. Li, B. Zou and S. X. Zhang, J. Am. Chem. Soc., 2015, 137, 931-939.

27 H. Ito, M. Muromoto, S. Kurenuma, S. Ishizaka, N. Kitamura, H. Sato and T. Seki, Nat. Commun., 2013, 4, 2009.

28 G. Q. Zhang, J. W. Lu, M. Sabat and C. Fraser, J. Am. Chem. Soc., 2010, 132, 2160-2162.

29 M. Tanioka, S. Kamino, A. Muranaka, Y. Ooyama, H. Ota, Y. Shirasaki, J. Horigome, M. Ueda, M. Uchiyama, D. Sawada and S. Enomoto, J. Am. Chem. Soc., 2015, 137, 6436-6439.

30 J. Wang, J. Mei, R. Hu, J. Z. Sun, A. Qin and B. Z. Tang, J. Am. Chem. Soc., 2012, 134, 9956-9966.

31 W. Z. Yuan, Y. Tan, Y. Gong, P. Lu, J. W. Lam, X. Y. Shen, C. Feng, H. H. Sung, Y. Lu, I. D. Williams, J. Z. Sun, Y. Zhang and B. Z. Tang, Adv. Mater., 2013, 25, 2837-2843.

32 Y. J. Zhang, Q. B. Song, K. Wang, W. G. Mao, F. Cao, J. W. Sun, L. L. Zhan, Y. K. Lv, Y. G. Ma, B. Zou and C. Zhang, J. Mater. Chem. C, 2015, 3, 3049-3054.

33 D. P. Yan and D. G. Evans, Mater. Horiz., 2014, 1, 46-57.

34 Z. Y. Ma, M. J. Teng, Z. J. Wang, S. Yang and X. R. Jia, Angew. Chem., Int. Ed., 2013, 52, 12268-12272.

35 S. K. Park, J. H. Kim, T. Ohto, R. Yamada, A. O. F. Jones, D. R. Whang, I. Cho, S. Oh, S. H. Hong, J. E. Kwon, J. H. Kim, Y. Olivier, R. Fischer, R. Resel, J. Gierschner, H. Tada and S. Y. Park, Adv. Mater., 2017, 29, 1701346.

36 P.-Z. Chen, J.-X. Wang, L.-Y. Niu, Y.-Z. Chen and Q.-Z. Yang, J. Mater. Chem. C, 2017, 5, 12538-12546.

37 S. Xu, T. Liu, Y. Mu, Y.-F. Wang, Z. Chi, C.-C. Lo, S. Liu, Y. Zhang, A. Lien and J. Xu, Angew. Chem., Int. Ed., 2015, 54, 874-878.

38 C. D. Dou, L. Han, S. S. Zhao, H. Y. Zhang and Y. Wang, J. Phys. Chem. Lett., 2011, 2, 666-670.

39 Z. L. Zhang, D. D. Yao, T. L. Zhou, H. Y. Zhang and Y. Wang, Chem. Commun., 2011, 47, 7782-7784.

40 Q. Benito, X. F. Le Goff, S. Maron, A. Fargues, A. Garcia, C. Martineau, F. Kahlal, S. Taulelle, T. Gacoin, J.-P. Boilot and S. Perruchas, J. Am. Chem. Soc., 2014, 136, 11311-11320.

41 X. Luo, J. N. Li, C. H. Li, L. P. Heng, Y. Q. Dong, Z. P. Liu, Z. Bo and B. Z. Tang, Adv. Mater., 2011, 23, 3261-3265.

42 J. Luo, L.-Y. Li, Y. L. Song and J. Pei, Chem. - Eur. J., 2011, 17, 10515-10519.

43 L. Wang, K. Wang, B. Zou, K. Q. Ye, H. Y. Zhang and Y. Wang, Adv. Mater., 2015, 27, 2918-2922.

44 K. Nagura, S. Saito, H. Yusa, H. Yamawaki, H. Fujihisa, H. Sato, Y. Shimoikeda and S. Yamaguchi, J. Am. Chem. Soc., 2013, 135, 10322-10325.

45 Y. Y. Qin, G. P. Li, T. Qi and H. Huang, Mater. Chem. Front., 2020, 4, 1554-1568. 
46 H. Nakayama, J.-i. Nishida, N. Takada, H. Sato and Y. Yamashita, Chem. Mater., 2012, 24, 671-676.

47 J. Nishida, H. Ohura, Y. Kita, H. Hasegawa, T. Kawase, N. Takada, H. Sato, Y. Sei and Y. Yamashita, J. Org. Chem., 2016, 81, 433-441.

48 J. A. Li, J. H. Zhou, Z. Mao, Z. L. Xie, Z. Yang, B. J. Xu, C. Liu, X. Chen, D. Y. Ren, H. Pan, G. Shi, Y. Zhang and Z. G. Chi, Angew. Chem., Int. Ed., 2018, 57, 6449-6453.

49 Z. Mao, Z. Yang, C. Xu, Z. Xie, L. Jiang, F. L. Gu, J. Zhao, Y. Zhang, M. P. Aldred and Z. G. Chi, Chem. Sci., 2019, 10, 7352-7357.

50 S. Y. Lee, T. Yasuda, Y. S. Yang, Q. Zhang and C. Adachi, Angew. Chem., Int. Ed., 2014, 53, 6402-6406.

51 P. Rajamalli, N. Senthilkumar, P. Gandeepan, P. Y. Gandeepan, P.-Y. Huang, M.-J. Huang, C.-Z. Ren-Wu, C.-Y. Yang, M.-J. Chiu, L.-K. Chu, H.-W. Lin and C.-H. Cheng, J. Am. Chem. Soc., 2016, 138, 628-634.

52 H. Tanaka, K. Shizu, H. Nakanotani and C. Adachi, J. Phys. Chem. C, 2014, 118, 15985-15994.

53 D.-G. Chen, T.-C. Lin, Y.-A. Chen, T.-C. Lin, Y.-T. Chen and P.-T. Chou, J. Phys. Chem. C, 2018, 122, 12215-12221.

54 K. Wang, Y.-Z. Shi, C.-J. Zheng, W. Liu, K. Liang, M. Zhang, H. Lin, S.-L. Tao, C.-S. Lee, X.-M. Ou and X.-H. Zhang, ACS Appl. Mater. Interfaces, 2018, 10, 31515-31525.

55 B. Li, Z. Li, F. Gou, J. Song, X. Jiang, Y. Wang, S. Gao, J. Wang, X. Pang, L. Zhao and Y. Zhang, ACS Appl. Mater. Interfaces, 2020, 12, 14233-14243.

56 J. F. Li, C. X. Hou, C. Huang, S. Q. Xu, X. L. Peng, Q. Qi, W.-Y. Lai and W. Huang, Research, 2020, 3839160.
57 J. F. Li, C. L. Yang, X. L. Peng, Y. Chen, Q. Qi, X. Y. Luo, W.-Y. Lai and W. Huang, J. Mater. Chem. C, 2018, 6, 19-28.

58 Y. Wan, J. F. Li, X. L. Peng, C. Huang, Q. Qi, W.-Y. Lai and W. Huang, RSC Adv., 2017, 7, 35543-35548.

59 Z. Yang, Y. Zhang, Z. Qiu, J. Zeng, J. Guo, S. Hu, Z. Zhao, X. Li, S. Ji, Y. Huo and S.-J. Su, ACS Appl. Mater. Interfaces, 2020, 12, 29528-29539.

60 J.-H. Tan, W.-C. Chen, S.-F. Ni, Z. Qiu, Y. Zhang, Z. Yang, C. Cao, Y. Huo and C.-S. Lee, J. Mater. Chem. C, 2020, 8, 8061-8068.

61 Y. Im, M. Kim, Y. J. Cho, J.-A. Seo, K. S. Yook and J. Y. Lee, Chem. Mater., 2017, 29, 1946-1963.

62 R. S. Nobuyasu, J. S. Ward, J. Gibson, B. A. Laidlaw, Z. J. Ren, P. Data, A. S. Batsanov, T. J. Penfold, M. R. Bryce and F. B. Dias, J. Mater. Chem. C, 2019, 7, 6672-6684.

63 C. Y. Wang, J. Zhang, G. K. Long, N. Aratani, H. Yamada, Y. Zhao and Q. C. Zhang, Angew. Chem., Int. Ed., 2015, 54, 6292-6296.

64 K. L. Wu, T. Zhang, L. Zhan, C. Zhong, S. L. Gong, Z. H. Lu and C. L. Yang, Adv. Opt. Mater., 2016, 4, 1558-1566.

65 R. Katoh, K. Suzuki, A. Furube, M. Kotani and K. Tokumaru, J. Phys. Chem. C, 2009, 113, 2961-2965.

66 G. He, L. L. Du, Y. Y. Gong, Y. L. Liu, C. B. Yu, C. Wei and W. Z. Yuan, ACS Omega, 2019, 4, 344-351.

67 H. S. Yuan, K. Wang, K. Yang, B. B. Liu and B. Zou, J. Phys. Chem. Lett., 2014, 5, 2968-2973.

68 J. P. Schmidtke, J. S. Kim, J. Gierschner, C. Silva and R. H. Friend, Phys. Rev. Lett., 2007, 99, 167401.

69 Y. Li, Z. Y. Ma, A. Li, W. Q. Xu, Y. C. Wang, H. Jiang, K. Wang, Y. S. Zhao and X. R. Jia, ACS Appl. Mater. Interfaces, 2017, 9, 8910-8918. 